\title{
Simultaneous X-ray radiography and diffraction topography imaging applied to silicon for defect analysis during melting and crystallisation
}

\author{
Maike Becker ${ }^{a}$, Gabrielle Regula ${ }^{a}$, Guillaume Reinhart ${ }^{a}$, Elodie Boller ${ }^{b}$, Jean-Paul Valade ${ }^{b}$, \\ Alexander Rack ${ }^{b}$, Paul Tafforeau ${ }^{b}$ and Nathalie Mangelinck-Noël ${ }^{a_{*}}$ \\ ${ }^{a}$ Aix Marseille Univ, Université de Toulon, CNRS, IM2NP, Marseille, France \\ ${ }^{b}$ ESRF - The European Synchrotron, CS40220, Grenoble Cedex 9, 38043, France \\ Correspondence email: nathalie.mangelinck@im2np.fr \\ Funding information : Agence Nationale de la Recherche (grant No. 14-CE05-0046-01, CrySaLID \\ project); Deutsche Forschungsgemeinschaft (scholarship No. BE 6627/1-1 to Maike Becker).
}

Synopsis A setup for simultaneous, time-resolved X-ray radiography and diffraction topography imaging is presented. It is used to study defect generation and growth mechanisms during heating, solidification and cooling of a silicon crystal.

Abstract One of the key issues to be resolved to improve the performance of silicon solar cells is to reduce crystalline defect formation and propagation during the growth process fabrication step. For this purpose, the generation of structural defects such as grain boundaries and dislocations in silicon must be understood and characterised. We combine in situ X-ray diffraction imaging, historically named topography, with radiography imaging to analyse the development of crystal defects before, during and after crystallisation. Two individual indirect detector systems are implemented to record simultaneously the crystal structure (topographs) and the solid-liquid morphology evolution (radiographs) at high temperature. This allows for a complete synchronisation of the images and for an increased image acquisition rate compared to previous studies that used X-ray sensitive films to record the topographs. The experiments are performed with X-ray synchrotron radiation at beamline ID19 at the European Synchrotron Radiation Facility (ESRF). We present in situ observations of the heating, melting, solidification and holding stages of silicon samples to demonstrate that with the upgraded setup detailed investigations of time-dependent phenomena are now possible. The motion of dislocations is recorded during the entire experiment, so that their interaction with grain boundaries and their multiplication through the activation of Frank-Read sources can be observed. Moreover, the capability to record with two camera-based detectors allows for the study of the relationship between strain distribution, twinning and nucleation events. In conclusion, the simultaneous recording of topographs and radiographs has great potential for further detailed investigations of the interaction and generation of grains and defects that influence the growth process and the final crystalline structure in silicon and other crystalline materials.

Keywords: X-ray radiography and topography; crystal growth; structural defects; silicon 


\section{Introduction}

In the field of silicon research for photovoltaic (PV) applications, real-time characterisation of the solidification process is crucial to understand the critical mechanisms producing defects during growth. Some types of defects can severely limit the conversion efficiency of solar cells by reducing the minority carrier lifetime (Oliveira et al., 2016, Woo et al., 2016, Wang et al., 1999, Fedotov et al., 1990). One of the most widely used silicon ingot fabrication method for solar cells is directional solidification. This manufacturing process usually leads to a polycrystalline ingot. The interaction of grains can cause new grain nucleation events affecting dislocation generation, propagation and multiplication (Tsoutsouva et al., 2016, Usami et al., 2010). In general, grain boundaries and dislocations can act as recombination sites for minority carriers. Especially the investigation of the interaction between grain boundaries and dislocations is important because dislocation bunches are very harmful for PV properties (Oriwol et al., 2017, Ryningen et al., 2011, Sopori et al., 2009). Thus, in order to enhance the PV properties of silicon wafers, systematic studies on the solidification of silicon are needed to reveal the mechanisms that produce dislocations, new grains and special types of grain boundaries that interact with dislocations.

To study crystal defects, X-ray diffraction imaging using white beam (topography) is widely used in the field of material sciences. Any deviation from the perfect plane orientation of the crystal structure, caused for example by dislocations, grain boundaries, or precipitates, results in a contrast (Raghothamachar et al., 2006, Wierzchowski et al., 2004). More precisely, the long range distortion field that is induced by the defects affects the intensity of the diffracted beam. In situ studies monitoring the heating, melting and/or solidification stages of silicon crystals have shown to give unique insights into the evolution of dislocations (Danilewsky et al., 2011, Vallino et al., 2001), cracks (Danilewsky et al., 2013) and grain competitions (Tsoutsouva et al., 2016). In these studies, the topography image was either recorded on high resolution $\mathrm{X}$-ray sensitive films or by a digital camera system i.e. indirect detection schemes compromising a camera lens-coupled to a scintillator screen (Hartmann et al., 1975, Tuomi et al., 1974, Chikawa \& Fujimoto, 1968). The camera option has the advantage of recording images with a very high time resolution (Rack et al., 2010). On the contrary, a film offers the advantage of a large field of view (FOV), wherefore multiple topographs (Laue spots) can be recorded at the same time (Oriwol et al., 2013). Depending on the research objective, one or the other option offers decisive advantages. In order to study the mobility of dislocations and strain fields, a camera-based system is preferred as it offers the required high temporal resolution.

In addition to topography imaging, the combination with other in situ techniques gives valuable supplementary information. Hänschke et al. (2017) combined topography with X-ray diffraction laminography to reconstruct the 3D pathways of dislocations in a silicon wafer. Tandjaoui et al. 
(2012) and Rack et al. (2016) combined topography with transmission imaging to characterise the growth of a silicon crystal and to measure the velocity of crack propagations in a silicon wafer, respectively. As Tandjaoui et al. (2012) and others (e.g. Tsoutsouva et al., 2016, Riberi-Béridot et al., 2015) used X-ray sensitive films to record the topographs alternately to X-ray radiography imaging during silicon solidification from the melt, Rack et al. (2016) used a high-speed camera system, which was necessary to follow the fast evolution of cracks in silicon wafers.

To study defect generation in silicon, the combination of alternating X-ray radiography and topography imaging has proven to be an excellent tool. Radiography complements the topography observations by providing information on growth rates and on the shape of the solid-liquid interface (Stamelou et al., 2017). Grain boundary grooves, generated at the encounter between the solidliquid interface and a grain boundary, reveal grain competition phenomena and the nucleation of new grains (Tandjaoui et al., 2013b). Apart from the scientific information, the real-time radiography image is also very important for controlling the experiment. In the live view, the liquid and the solid phases are distinguishable mainly due to their regular and irregular shapes, respectively, so that the height of the solid liquid interface, the shape of the liquid sample and possible voids can be identified. It allows taking corrective action during the in situ experiment which is very important for such a long experiment (12 hours in total) and limited beam time.

The previous in situ solidification studies mentioned in the above paragraph, used alternating X-ray radiography and topography imaging recording the topographs on X-ray sensitive films with limited film exposure rates of $0.04 \mathrm{~Hz}$ (Tandjaoui et al., 2013a). The limitation results from the complicated handling of the films as well as from the time consuming post-processing. Although these experimental results allowed elucidating and will continue to contribute to the understanding of many fundamental growth phenomena, important defect interactions and movements were actually missed because of the limited acquisition rate of the X-ray topography images. Thus, highly dynamic processes like dislocation motion were not accessible. Moreover, the simultaneous recording of radiographs and topographs was not possible which limited the investigation of the correlation between new grain nucleation (observed in the radiographs) and changing strain fields (observed in the topographs). Consequently, to study more rigorously dislocation interactions, strain (re)distribution, defect dynamics and competition, we have extended the experimental setup using two camera systems to record both, topographs and radiographs at the same time. Thus, the recording frequency of the topography images increases by more than one order of magnitude and a fully synchronised radiography and topography imaging is obtained allowing direct comparison. The enhancement of the recording frequency now provides the opportunity to study the propagation, multiplication and rearrangement of dislocations by interaction with themselves, grain boundaries 
and the solid-liquid interface, during the entire experiment. We discuss the new opportunities arising with this imaging setup for the characterisation of crystal structures using three examples that include heating, melting, solidification and holding stages of a silicon crystal inside a solidification furnace imposing a temperature gradient.

\section{Experimental}

\section{X-ray radiography and topography setup}

The experiments were carried out at the European Synchrotron Radiation Facility (ESRF) at beam line ID19. The beamline is ideally suited for this experiment because of the large field of view and the excellent and uniform flux of photons. The long source-to-sample distance of $145 \mathrm{~m}$ provides a (partial) coherent beam. A beam current of $200 \mathrm{~mA}$, a Wiggler gap of $61 \mathrm{~mm}$ and a $0.7 \mathrm{~mm}$ Al filter were used. The whole experimental setup is schematically shown in Figure 1. When the white beam illuminates the $300 \mu \mathrm{m}$-thin silicon sample inside the solidification furnace, the beam diffracts according to Bragg's law generating a Laue diffraction pattern with several diffraction spots related to specific lattice planes. A scientific CMOS camera lens-coupled to a LuAG scintillator (commercial Ce-doped $\mathrm{Lu}_{3} \mathrm{Al}_{5} \mathrm{O}_{12}$, Crytur company - Czech Republic) was used to record the images of one of the diffraction spots (topography) in transmission Laue geometry. The camera records $2048 \times 2048$ pixels with a nominal pixel size of $6.5 \mu^{2}$ and a dynamic range of 16 bit. It was coupled with a $\times 1.5$ optic to decrease the pixel size to $4.3 \mu^{2}$. The camera was mounted to a manually on air pads moveable rack positioned around one meter away from the sample. To fine-tune the camera position, the rack was equipped with two automatic motion systems that allowed the camera to be moved horizontally (left and right) and vertically in increments as fine as $20 \mu \mathrm{m}$. This enabled the diffracted spot to be positioned exactly in the middle of the camera from the control room. The position of the camera is limited to an area above and to the right side (when looking in the same direction as the X-ray beam) of the primary beam. This is due to the mounting system located on the right side of the setup and due to the fact that the camera must not block the primary beam. Moreover, lack of space inside the experimental hutch restricted the mounting of the camera system to the right side. The available area for topography recording is indicated in Figure 1 by the grey striped region in the top view image.

The primary beam passing through the sample is used to record the radiography images. To this end, the white beam is turned monochromatic at a target energy of $17.5 \mathrm{keV}$ using a vertically diffracting Si (111) double-crystal monochromator. The use of monochromatic light is essential to exploit the weak attenuation contrast originating from the density difference between the solid $\left(2.31 \mathrm{~g} \mathrm{~cm}^{-3}\right)$ and the liquid $\left(2.56 \mathrm{~g} \mathrm{~cm}^{-3}\right)$ silicon phases in the radiography images for which the contrast is based 
on absorption variations. The radiography image was recorded using a second detector (sCMOS lenscoupled to a LuAG scintillator) positioned around $7 \mathrm{~m}$ downstream to also benefit from propagation phase contrast imaging (edge detection mode). The camera records $2048 \times 2048$ pixels with a nominal pixel size of $6.5 \mu \mathrm{m}^{2}$ and a 16 bit dynamic range.

Images recorded from both modes are fully synchronised. The image acquisition rate was at maximum 2 frames per second which is sufficient to follow the solidification front of the samples. Shorter recording times may be realized in future experiments, but due to the long experimental times of several hours which includes heating, melting, solidification, and cooling, a compromise between acquisition rate and stored data volume must be found.

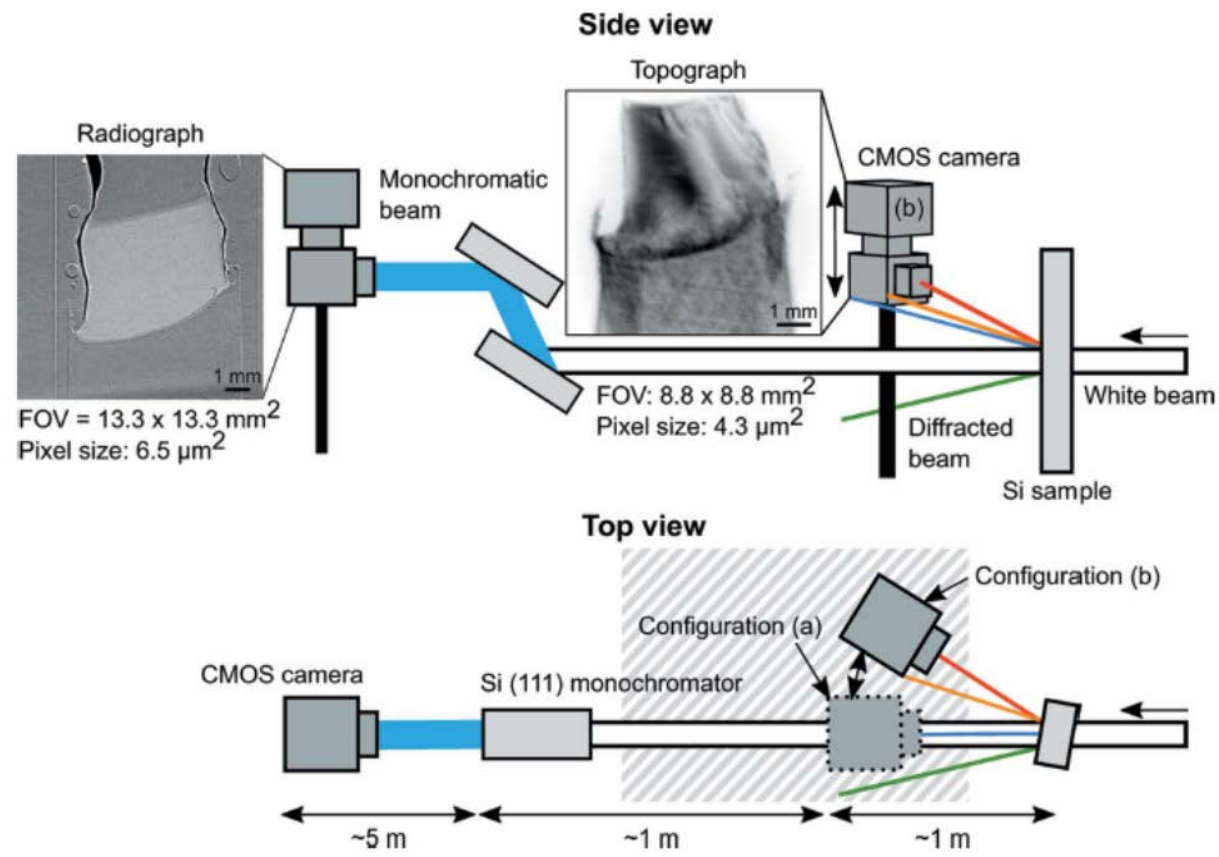

Figure 1: Schematic drawing of the experimental setup using X-ray radiography and diffraction topography modes simultaneously to monitor silicon solidification. The incident X-ray white beam comes from the right-hand side and hits the silicon sample that is positioned inside a solidification furnace not shown here. The grey striped area shows the possible positions of the topography camera. (a) and (b) mark two of the possible camera configurations.

The choice of the diffraction vector of the recorded spot and the alignment of the camera with respect to the sample face is an important aspect because it influences the appearance and the information that can be revealed from the recorded topography images. Ideally a spot with a high intensity induced by the crystal plane structure factor should be chosen to better reveal defects. 
The larger the angle is between the normal of the sample surface and the selected diffraction spot, the more of the thickness of the sample becomes visible because of the projection effect. The thickness of the sample is evidenced through a light grey contrast zone at the sample edges and at twin boundaries when neighbouring grains do not diffract with the same diffraction vector. However, as the sample is only $300 \mu \mathrm{m}$ thin, the projection effect is rather small.

When the recorded topograph is positioned just above the primary beam as in configuration (a) shown in Figure 1 and Figure 2, the effect of the projection only affects the vertical direction, so that the thickness of the sample is visible in the upper and lower parts. Therefore, more details of the solid-liquid interface become observable. The travel length of the diffracted beam is the same at all positions, so that the image is not distorted. If the camera optics do not face the diffracted beam directly, as it is shown for configuration (b) in Figure 1 and Figure 2, an additional geometrical effect appears in the images. As an example, in (b) the diffracted beam travels from the sample to the right and upwards. In combination with a tilted camera screen, the travel length of the beam before it hits the camera varies from the left to the right side of the image (from $d_{1}$ to $d_{3}$ ). Therefore, the diffracted light arrives later at the left upper side of the camera $\left(d_{1}\right)$ than at the right lower side $\left(d_{3}\right)$. This affects the whole image by changing for example horizontal lines in the sample in diagonal downward tilted lines in the images. Additionally, the width of the image (w) is compressed with respect to the real width of the sample $(w+\Delta w)$. Both camera alignments (a) and (b) were used in the experiments discussed in the following.

Configuration (a)
Configuration (b)

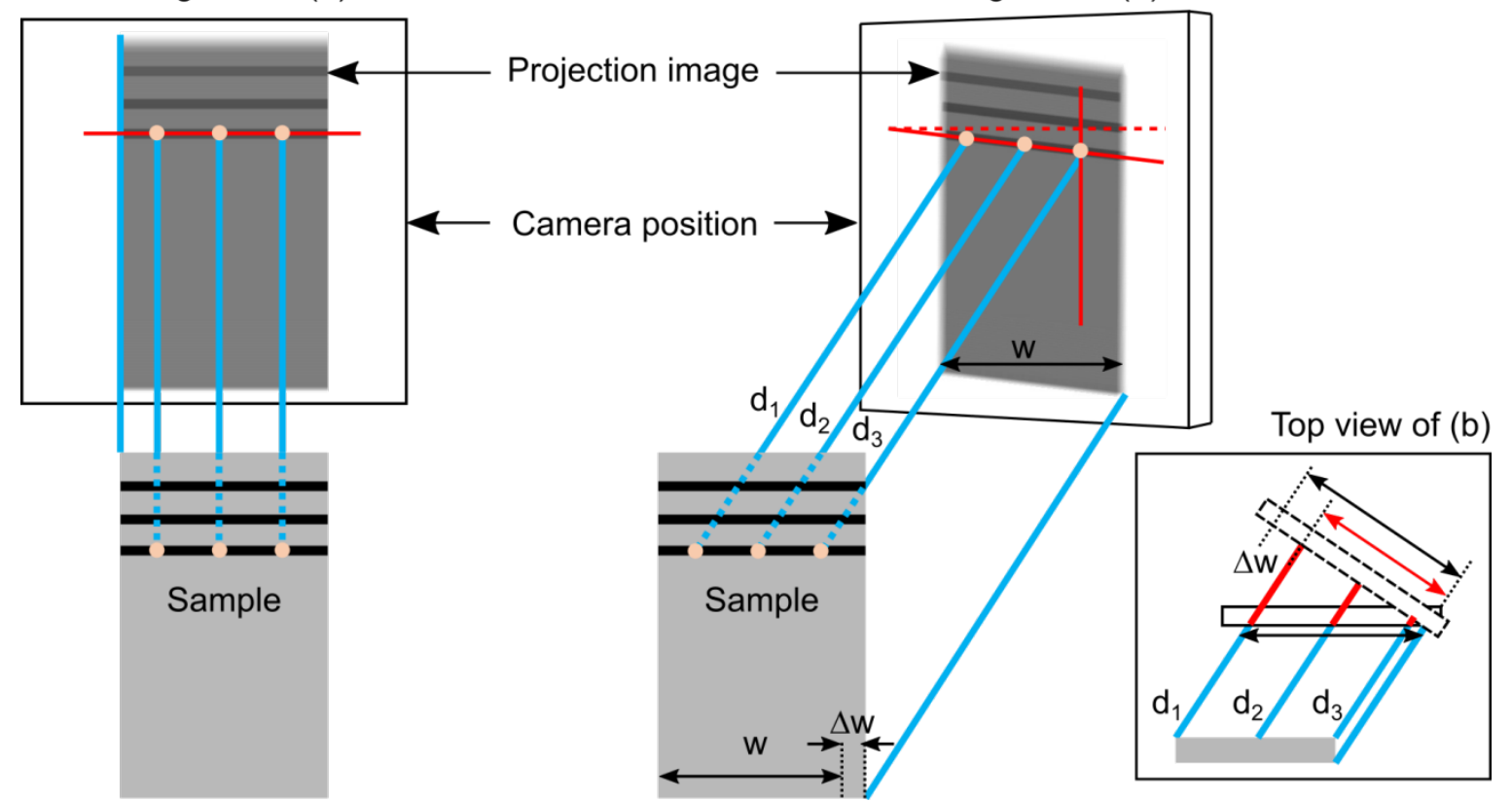

Figure 2: Schematic drawing of the projection effect of the camera image. In configuration (a), the camera is positioned above the primary beam. The camera surface is aligned perpendicular to the 
primary beam, thus the image is not compressed. In configuration (b) the camera is positioned at the upper right of the primary beam and the camera surface is inclined. Hence, lines that are horizontal in the sample are projected as downward tilted lines on the image and the sample image is compressed by $\Delta \mathrm{w}$ as illustrated in the top view of (b).

\section{In situ silicon solidification experiments}

An additional main originality of this work is the combination of the advanced X-ray imaging setup described above with a solidification high temperature gradient furnace for controlled solidification. The Bridgman furnace used is named GaTSBI (Growth at high Temperature observed by Synchrotron Beam Imaging) and was designed to be X-ray transparent and to reach temperatures up to $2073 \mathrm{~K}$ under high vacuum conditions. A more detailed description of the furnace can be found elsewhere (Tandjaoui et al., 2012). As the beam must pass the entry and exit windows of the furnace that are made out of $0.5 \mathrm{~mm}$ thick aluminium, and through $2 \mathrm{~mm}$ of vitreous carbon insulation, a high photon flux is needed for good quality imaging.

The experiments were carried out on single-crystalline silicon samples having two different crystallographic orientations as shown in Figure 3. The samples, provided by SIL'TRONIX Silicon Technologies, were produced with $9 \mathrm{~N}$ material by the float-zone technique and contain no visible dislocations at the beginning of the experiments. Oxygen and carbon concentrations are below $<10^{15}$ at $\mathrm{cm}^{-3}$ and metallic impurity contamination is limited to $10^{11}$ at $\mathrm{cm}^{-3}$. Two thin boron nitride (BN) plates serve as crucible material. One of the BN plates contains a slot of the size of the sample in which it is placed. The two BN plates are held together from the outside by two Molybdenum clips. Thus, a slight pressure acts on the main surfaces of the samples.

To conduct a solidification experiment, the crucible is positioned in the gradient furnace. The main surface of the sample (here the (110) plane) was tilted from the primary beam by about $5^{\circ}$. This rotation was necessary to bring some of the low indexed crystal planes into Bragg diffraction condition. A $180^{\circ}$ rotation of the sample around the $x-, y$ - or z-axis would result in the same diffraction pattern. As the exact orientation of the sample is unknown, we use in the following the orientation notation sketched in Figure 3. A temperature gradient was imposed on the sample by applying different temperatures to the upper $\left(T_{T}\right)$ and lower $\left(T_{B}\right)$ heaters. The temperature was slowly increased to obtain partial melting of the Si sample from the top $\left(\mathrm{T}_{\text {liq }}=1687 \mathrm{~K}\right)$. Thanks to the in situ recording, the movement of the solid-liquid interface through the FOV can be observed. This allows to position the solid-liquid interface in the lower third of the FOV by regulating the temperature. Solidification from the remaining seed was triggered by applying a constant cooling 
rate (from 0.2 up to $4 \mathrm{~K} \mathrm{~min}^{-1}$ ) to both heaters, so that the liquid silicon directionally solidified from bottom up.

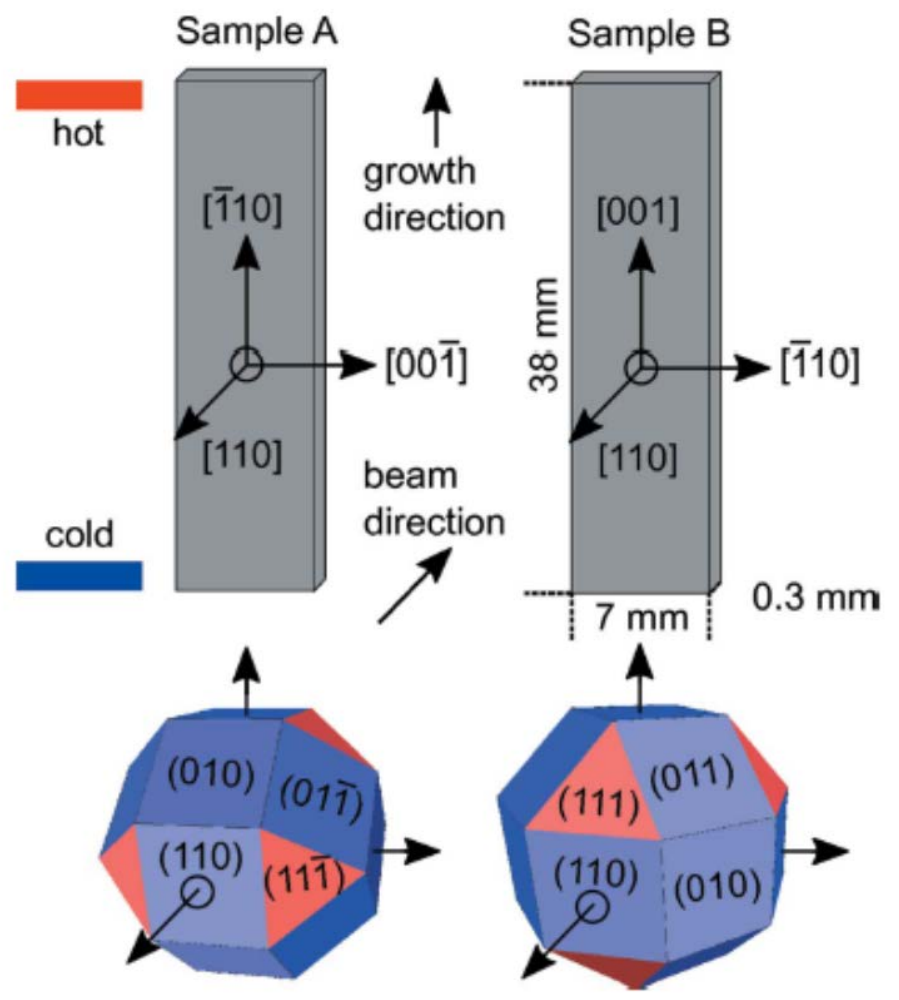

Figure 3: Drawing of the dimensions and of the crystallographic orientations of the silicon samples A and $\mathrm{B}$ used for the experiments.

\section{Results and Discussion}

\section{Evaluation of topography imaging techniques}

Topographs were recorded using a camera $\left(8.8 \times 8.8 \mathrm{~mm}^{2}\right.$ maximum FOV) and for sample A using additionally a fine-grained X-ray sensitive film (AGFA Structurix D3-SC, $176 \times 125 \mathrm{~mm}^{2}$ FOV). Topography images of sample A recorded with a film and with the camera are shown in Figure 4 (ad) and in Figure 4 (e), respectively. The camera image shows a topograph that was recorded shortly after solidification was completed in the FOV. The film was exposed only a few minutes later when the sample had further cooled down by $\sim 10 \mathrm{~K}$. The exposure times for the film and for the camera were $0.4 \mathrm{~s}$ and $0.5 \mathrm{~s}$, respectively. Like the film, the camera surface was also aligned perpendicular to the primary beam, not far from configuration (b) shown in Figure 2. Therefore, both recording techniques are comparable.

The film shown in Figure 4 (a) displays six different diffraction spots. Knowing the original sample crystallographic orientation, the crystallographic planes corresponding to the diffraction spots could 
be identified. Due to the polycrystalline nature of the solidified sample, crystals having different orientations can be identified on different spots. Analysing several diffraction spots is important as dislocations can be extinguished or weakened on certain spots depending on their Burgers vector $\vec{b}$. Dislocations are invisible on a particular diffraction spot if their Burgers vector $\vec{b}$ is perpendicular to the diffraction vector $\vec{g}(\vec{b} \cdot \vec{g}=0)$. Therefore, as with the camera-based system only one spot can be observed over the entire experiment, the selection of the spot for the camera imaging is very important. For example, by comparing the diffractions spots in Figure $4(b-d)$, different grains can be identified. The spots [113] and [004] reveal twin relationships of some grains on the left side, which

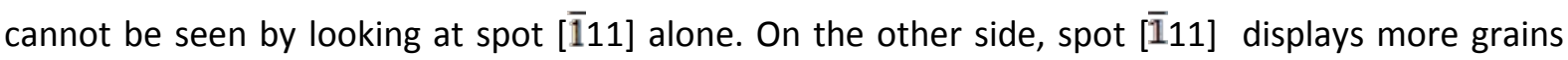
than the other spots indicating that these grains have a common diffraction vector $\vec{g}[\overline{1} 11]$. The dislocations are probably standard $0^{\circ}$ or $60^{\circ}$ dislocations with Burgers vector $b=a / 2\langle 110\rangle$ and line direction $\langle 110\rangle$, where $a$ is the lattice constant. Dissociation into two $1 / 6<112\rangle$ Shockley partials separated by an intrinsic stacking fault is likely to occur, but cannot be resolved with the here used $\mathrm{X}$-ray topographic technique. During the motion of dislocations, their character can change along the dislocation line. The exact Burgers vector cannot be determined from Figure 4, since all recorded diffraction vectors lie in one zone axis. Dislocations with Burgers vectors perpendicular to the zone axis, of which there are six, are in an extinction position. Thus, there are six possible Burgers vectors left for the visible dislocations. 
(a)

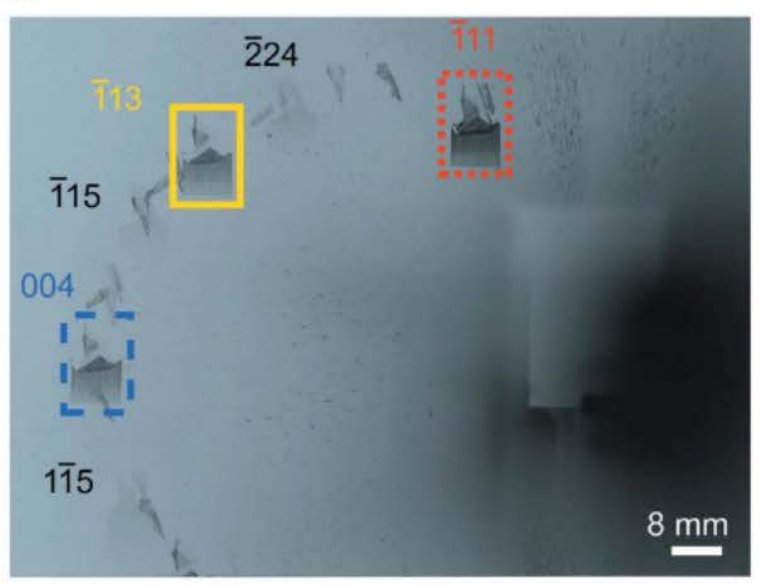

(b)

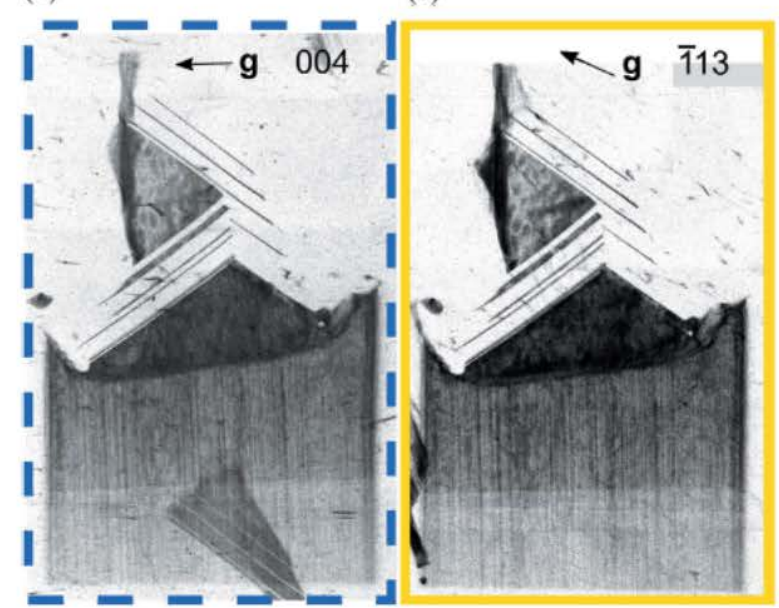

(d)

$(e)$
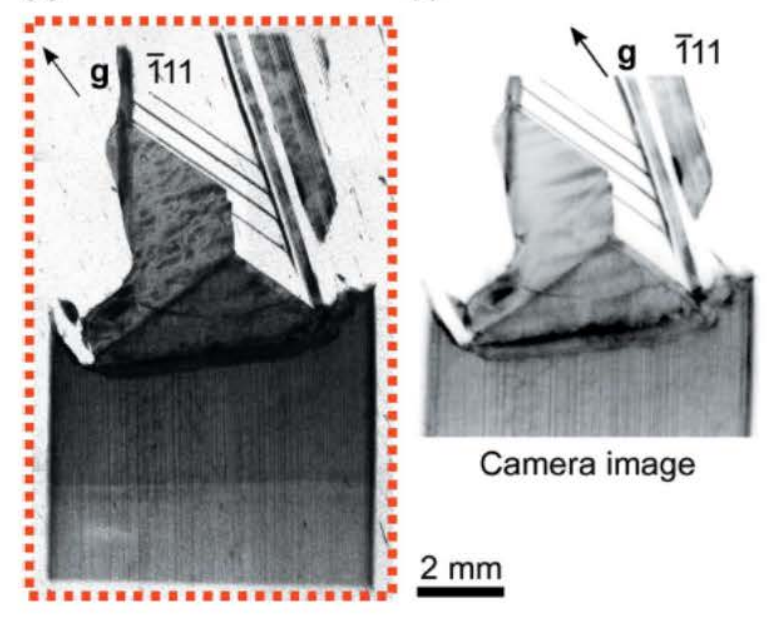

Figure 4: Topography images of sample A recorded shortly after solidification was completed in the FOV. The sample was solidified by lowering the temperature by $1 \mathrm{~K} \mathrm{~min}^{-1}$ in an applied temperature gradient of $3 \mathrm{~K} \mathrm{~mm}^{-1}$. (a) Diffraction pattern recorded on an X-ray sensitive film. The numerous small dark speckles on the background are caused by an additional Al filter and do not originate from the sample. (b-d) Close-ups of the three topographs corresponding to the diffraction vectors $\overline{1} 11, \overline{1} 13$ and 
004, respectively. (e) Topograph of the $\overline{1} 11$ diffraction spot recorded with the camera-based detection system.

Figure 5 shows two close-ups of the same $\overline{1} 11$ spot recorded with (a) the camera and with (b) the Xray sensitive film. The topographs on the film were digitalized with an optical microscope equipped with a camera using a magnification lens of $\times 2.5$. On closer inspection, small differences are noticeable. On the one hand, features can be better distinguished on the film (blue arrows 1) because its spatial resolution $(5 \mu \mathrm{m})$ is better than the resolution of the camera $(9 \mu \mathrm{m})$. This is only a technological issue due to the fact that large field of view cameras have not yet reached the spatial resolution of sensitive films. On the other hand, a connected dislocation network in the lower crystal is clearly visible on image (a), but not on image (b) (white arrows 2). These dislocations originate from the seed grain and have propagated along with the solid-liquid interface during growth. It is clearly visible on the camera image that most of these dislocations pile-up at the diagonal twin grain boundary. Furthermore, faint upward lines on the left grain are again more clearly visible in (a) than in (b) (red arrows 3). Apparently, the contrast caused by distortions is greater on the camera images than on the films. This is related to the higher dynamic range of the camera compared to the film which was discussed in detail by Danilewsky et al. (2008).

Besides, additional blurring on the camera image is introduced by the inclined angle of beam incidence (not perpendicular) on the scintillator screen. The incidence angle of the (1111) spot is $2 \theta_{B}=5.8^{\circ}$ with respect to the primary beam, where $\theta_{B}$ is the Bragg angle. The incoming photons have an energy of $\sim 39 \mathrm{keV}$, which corresponds to a length of half-absorption of $120 \mu \mathrm{m}$ for the LuAG scintillator. It basically means that the whole thickness is crossed by the beam still producing light. Considering the $5.8^{\circ}$ intercept angle with the scintillator and the scintillator thickness of $500 \mu \mathrm{m}$, the photon path laterally covers a width of $51 \mu \mathrm{m}$ in the scintillator leading to an activation of 12 adjacent pixels by the same photon signal along an axis oriented at $28.2^{\circ}$ from the vertical. Accordingly, the blurring and its impact on the image quality due to the depth of penetration of X-rays in the LuAG scintillator is significant. In addition, as the blurring has a direction (is not isotropic) due to the incidence angle, it can be considered as a motion blurring enhancing structures that are oriented along the blurring direction. This explains why dislocations aligned top left can be better distinguished on the camera image than on the X-ray film, where this effect does not occur.

All in all, a film offers the advantage of a large FOV, whereas a camera provides a greater contrast around defects (a higher dynamic range), a significant higher acquisition rate and a more comfortable handling. The choice which of both recording options to use depends on whether it is more important for the study of interest to have a larger FOV (several topographs) or a higher time resolution. As we want to study defect interactions, a high acquisition time is necessary. However, films can be 
additionally used on a one-off basis to provide important complementary information from different $\vec{g}$ vectors.

(a)

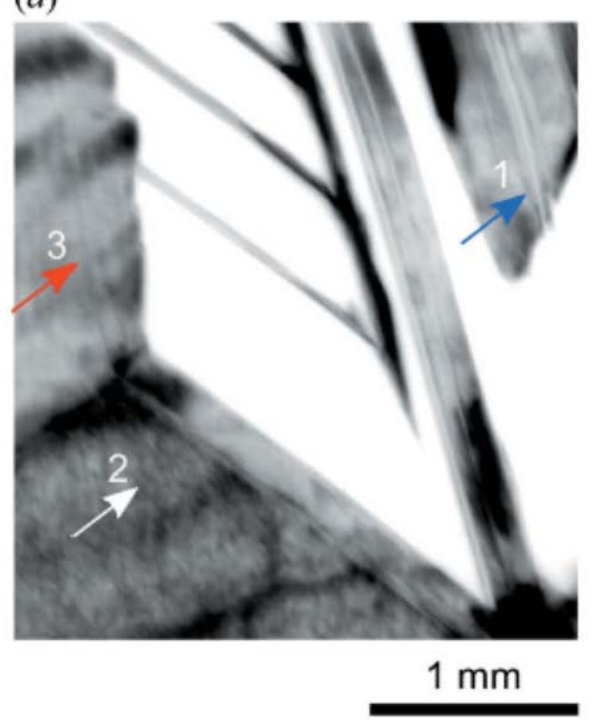

(b)

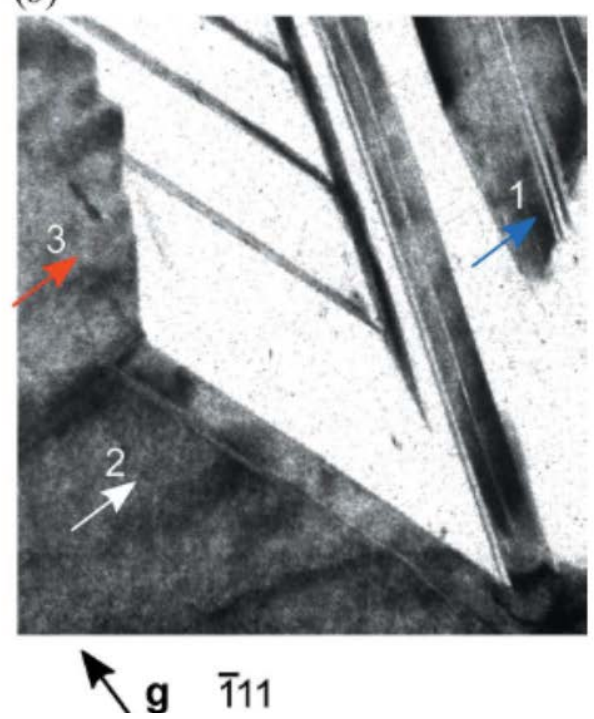

Figure 5: Comparison of the same topograph corresponding to the $\overline{1} 11$ spot recorded with (a) a camera-based system and (b) an X-ray sensitive film.

\section{Heating and Melting}

During the heating and melting stages of sample B, the movement of individual dislocations and their self-organization was observed. Dislocations induce a contrast in the homogenous background because local planes near a dislocation core are bent. If these planes are tilted into a diffracting orientation, the intensity of the diffracted beam is increased which is why they appear as darker lines. If the dislocation density exceeds $\sim 10^{5} \mathrm{~cm}^{-2}$, individual dislocations can no longer be resolved and the entire area appears dark. Note that the dislocations can also appear brighter than the background, when due to sample warping or bowing the corresponding crystal planes reflect at an angle far away from their surrounding planes (George, 1977).

It was shown by Tsoutsouva et al. (2018) that at a temperature of $1373 \mathrm{~K}$ a horizontal slip system is activated for a sample of the same orientation as sample B and with the same crucible arrangement and comparable temperature conditions. It was concluded that this slip system initiates from the sample side damaged zones during preparation and is activated by an external force acting on the sample surface, i.e. perpendicular to the sample thickness. As can be seen in Figure 6 (b) and (c), the same horizontal $\{111\}$ slip system was activated in sample B. The dislocations span the entire width of the sample as straight long segments. It shows that even at temperature close to the melting point, in covalent materials like silicon, the Peierls-Nabarro potential remains strong enough to keep 
the dislocation lines along $\langle 110\rangle$ directions. The fact that the segments are not horizontal and parallel to the $<110>$ direction, but tilted by an angle of $8^{\circ}$ downwards from left to right, is due to the tilted camera orientation in this experiment as explained in section 2.1. Although the dislocation density in the seed is rather high (but not beyond $10^{5} \mathrm{~cm}^{-2}$ ) and the local stress is low (estimated weaker than $500 \mathrm{kPa}$ ), some dislocations are still mobile. The distinct motion is probably caused by the high temperature close to the melting point, and favoured by the small sample thickness allowing dislocations to reach the sample surface before they can interact with each other via crossslips which would induce the formation of jogs and Frank-Read sources.

In this sample, the dislocations preferentially propagate from the left to the right side which is highlighted by a white arrow in the middle row of Figure 6 (b). This may be due to the isotherms inclined from right to left as can be inferred when looking at the melting interface (see synchronised radiography images in Figure 6 (a)). The inclination of the interface indicates that a transverse temperature gradient from right to left exists. A video of the dislocation propagation, highlighted in the image sequence of Figure 6 (c), is available as supplementary material.

When the solid-liquid interface is within the FOV, a clear change in the dislocation network is observed close to the interface. It is reflected in the movement of dislocations in diagonal glide planes. The white lines serve as a guide for the eyes to observe the direction of dislocation movement. When melting starts, a new glide plane is activated close to the interface. The dislocations that propagate on this new glide plane are created either by new sources that originate at the solid-liquid interface, by existing tiny dislocation loops that can expand due to higher local stresses or by both phenomena at the same time. 


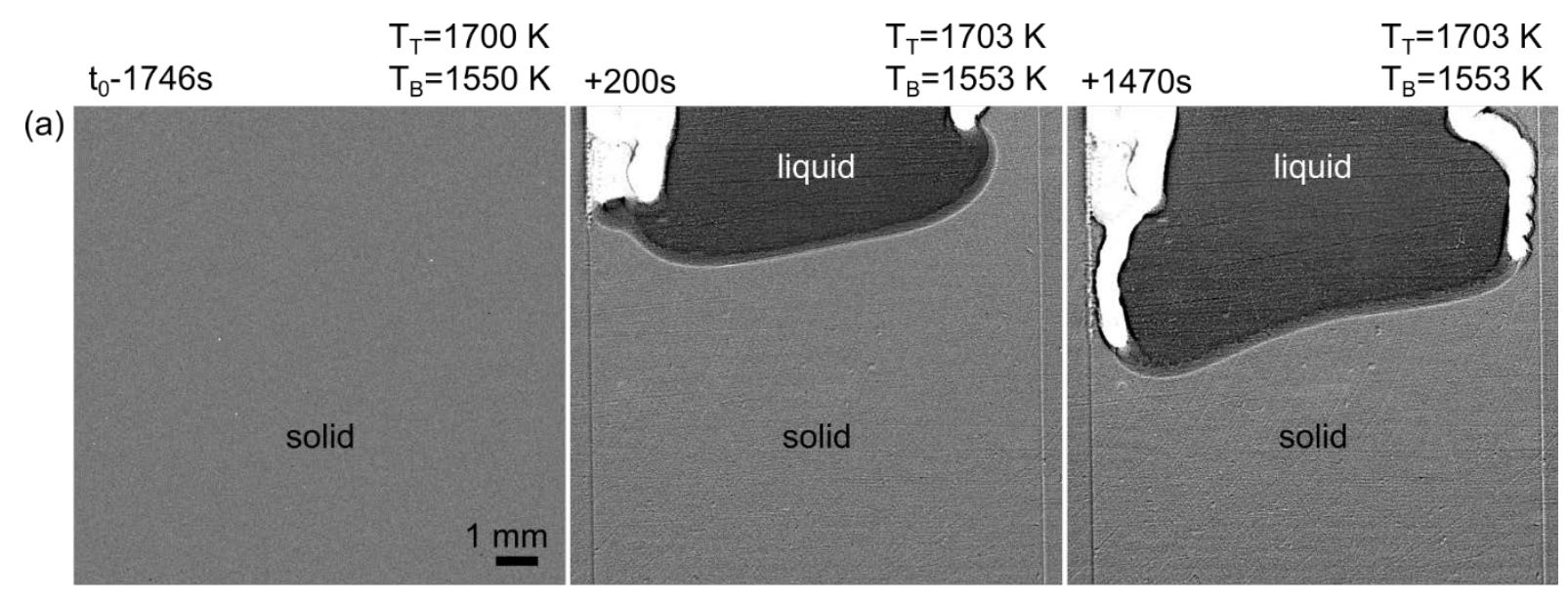

(b)
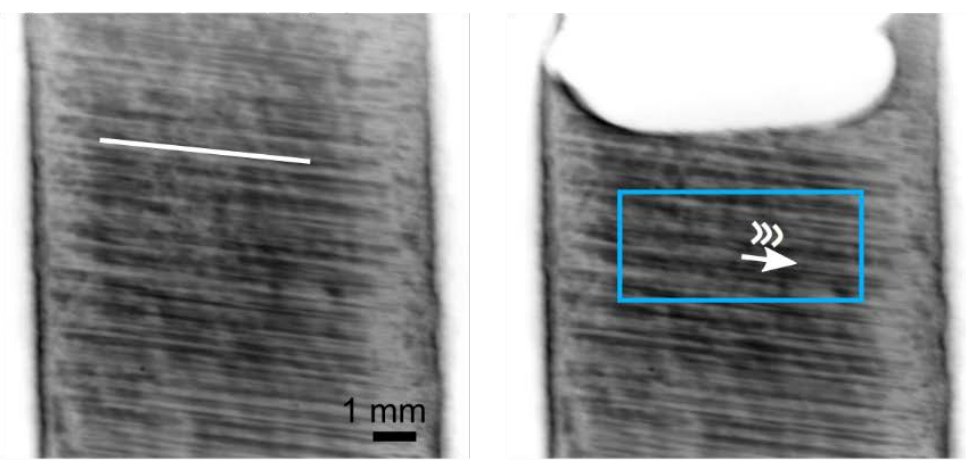

$\mathrm{t}_{0}-1557 \mathrm{~s}$
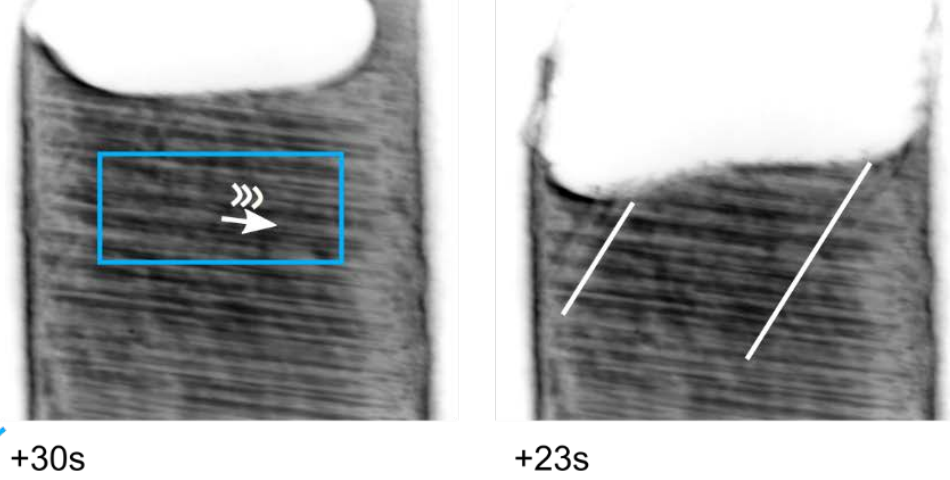

$+23 s$

(c)

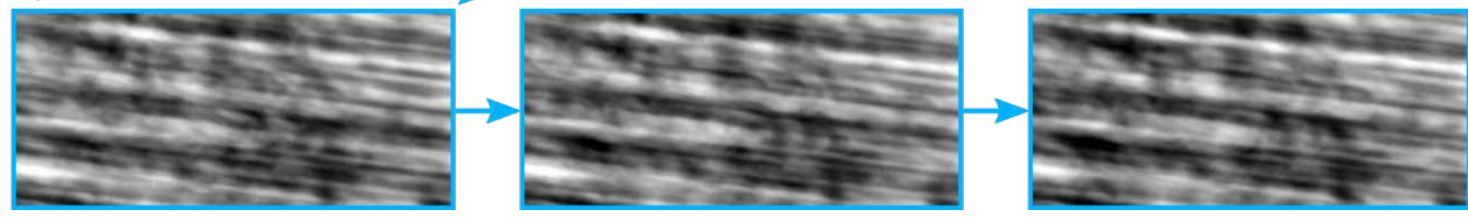

Figure 6: Sequence showing synchronised (a) radiography and (b) topography images of the melting of silicon sample B. Flat field image processing was applied to the radiography images to highlight the solid-liquid interface. (c) shows a zoom of the topography images corresponding to the blue rectangle in (b). A video is available as supplementary material to show the propagation of dislocations in more detail. $t_{0}$ corresponds to the starting time of solidification and $T_{T}$ and $T_{B}$ to the temperatures of the top and the bottom heaters, respectively. The heating rate is $2 \mathrm{~K} \mathrm{~min}^{-1}$.

\section{Solidification}

During solidification, radiography and topography images can be observed simultaneously. Figure 7 follows a twinning and a nucleation event at the solid-liquid-vapour boundary during solidification of sample B. The facet of the deep groove in (a) corresponds to a $\{111\}$ plane. The angle between this facet and the horizontal is $\sim 54^{\circ}$ which corresponds to the angle between $\{111\}$ and $\{100\}$ crystallographic planes. The blue arrows $(1,2)$ in Figure $7(a)$ and $(b)$ point to twinning events on the facet. Twinning events can be identified by white areas in the processed radiography images that indicate abrupt growth at the solid-liquid interface. Considering what was also observed in previous works during the solidification of single-crystal seeded samples (Tsoutsouva et al., 2016, Riberi- 
Béridot et al., 2015), new grains nucleate always with a $\Sigma 3\{111\}$ twin relationship. Nucleation of twins preferentially occurs at the sample boundaries on $\{111\}$ facets because $\Sigma 3$ twinning on $\{111\}$ planes is very common for silicon as it is associated to a very low surface energy (Duffar, 2010).

By comparing the diffraction images and the radiographs, the relative change of strain in the region of interest before and after the twinning event is investigated. The close-ups of the topography images (a) and (b) (bottom row) show that there is no significant strain on the selected diffraction spot near the grain boundary before the diagonal twinning occurs. In the radiography image (c) (top row), a nucleation event takes place on the side of the sample. This grain (I) is not visible on the corresponding synchronised diffraction image (c) (bottom row), which shows that this new grain has a different crystallographic orientation without common planes diffracting with the same Bragg angle on the selected diffraction spot. When the diffraction vector $\vec{g}[\mathrm{hkl}]$ of the new grain is a common diffraction vector with the seed grain, both grains are visible on a common diffraction spot. Thus, depending on the orientation relationship of the newly nucleated grain compared to the seed grain, and depending on the diffraction spot selected for topography, new grains are either visible or not visible on the selected diffraction spot. The fast growth of the new grain indicates that the undercooling at the bottom of the groove is higher compared to the average undercooling at the solid-liquid interface.

Compared to the previous images, the strain at the location next to the new nucleation increases (black arrow (3)). In (d) (bottom row) the strain level has increased even more (darker contrast at black arrow (4)), which is attributed to the nucleation of a new neighbour grain. Higher strain levels at grain boundaries differing from $\Sigma 3$ type grain boundaries have also been reported in previous works (Tsoutsouva et al., 2016), although the strain building could not be followed step by step with the former time resolution. The observed accumulation of strain can be attributed to two concomitant mechanisms. First, the lower perfection of the crystalline arrangement of the grain boundary when differing from a $\Sigma 3$ is at the origin of local crystalline network distortions and/or dislocation emissions. Second, the rapid grain growth makes the new nucleated grain to enter in competition and most likely to generate stress among its neighbour grains. 

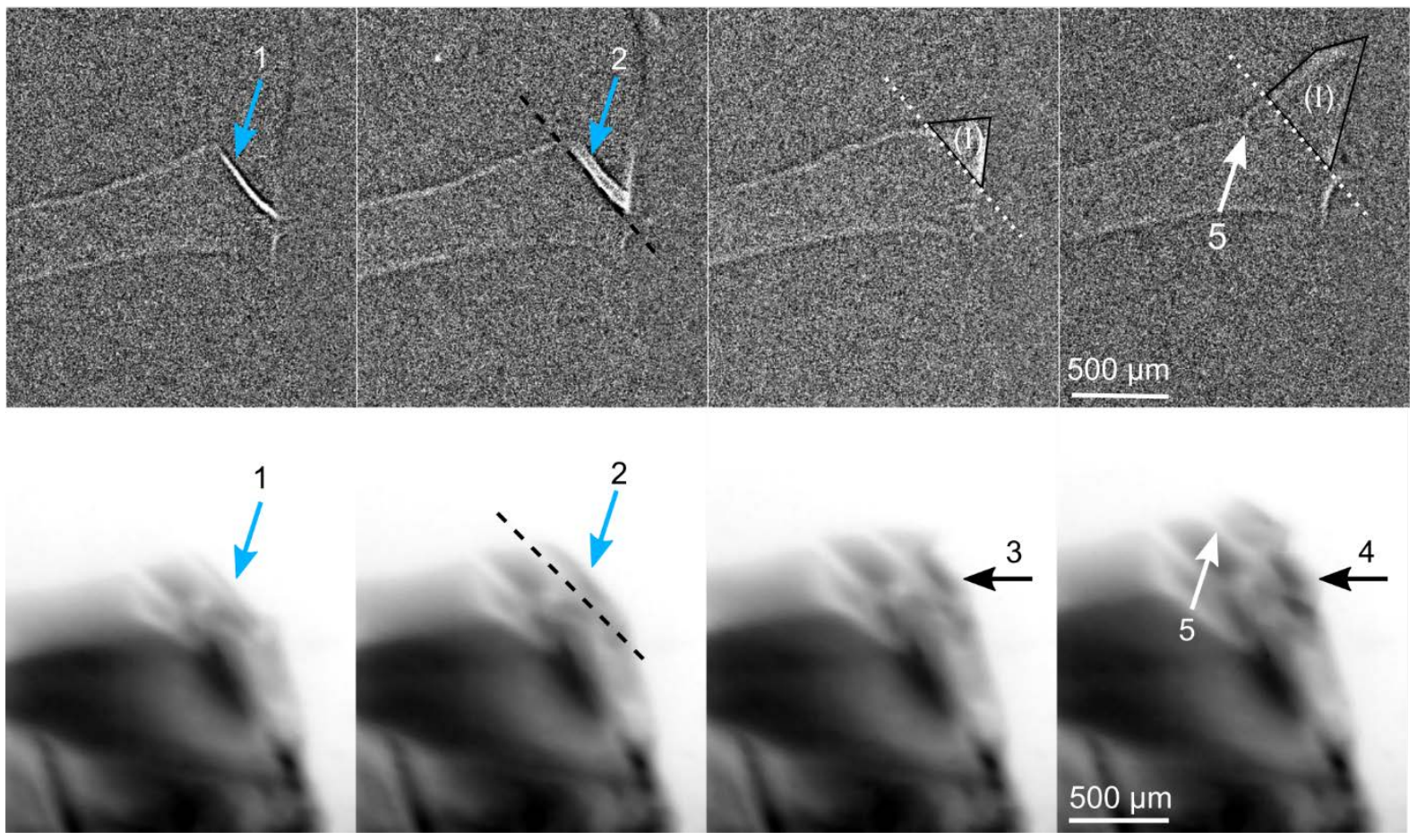

Figure 7: Time-resolved radiography (top row) and topography (bottom row) sequences of twinning and nucleation events at the right side of sample B. The blue arrows $(1,2)$ indicate the twinning events in (a) and (b). The black arrows in the topography images $(3,4)$ mark the same height and indicate the area where increased strain is observed. The white arrow (5) points to a grain boundary groove in (d) that develops after the new grain nucleation. To make the solid-liquid interface visible, the radiography images were processed using successive image division (cf. Tandjaoui et al., 2013a). $\mathrm{t}_{0}$ corresponds to the starting time of solidification.

\section{Holding in a temperature gradient at high temperatures}

After solidification of sample B, the crystal was kept in a stable temperature gradient for 1.5 hours. The temperature of the top and bottom heaters were $1693 \mathrm{~K}$ and $1543 \mathrm{~K}$, respectively. The effective temperature gradient acting on the sample is lower than the applied gradient as discussed in Stamelou et al. (2017) and was in the order of $\mathrm{G}=1 \mathrm{~K} \mathrm{~mm}^{-1}$ for the particular experiment shown here. During the holding stage, local dislocation rearrangement and strain relaxation were observed. In several grains, dislocation multiplication occurred by the activation of Frank-Read sources as it is shown by red arrows in Figure 8 (a video is available in the supplementary material). Multiple sources formed during the recorded timespan of more than 1 hour. The measured radii of the semicircles are in the range of $\mathrm{R}=60-80 \mu \mathrm{m}$. Due to the spot projection and the fact that the particular $\{111\}$ plane on which the dislocations propagate is not known, the loops are probably not parallel to the camera surface and, thus, the radii are overestimated. However, as the loops propagate almost 
as circles, we assume that the length measurement of the semi-circles are sufficiently reliable for a stress evaluation.

The shear stress $\sigma$ required to bend a dislocation to a radius $R$ is given by $\sigma=\mu b / R$, where $\mu=64 \mathrm{GPa}$ is the shear modulus of silicon (Wortman \& Evans, 1965) and $b=a / 2<110\rangle$ is the Burgers vector of $a$ perfect dislocation in silicon with $a=0.543 \mathrm{~nm}$ the lattice constant of silicon. As the constants are given for room temperature, but the experiment takes place close to the melting point, the shear stress is only an estimate. Consequently, the corresponding calculated shear stress is in the order of 200-300 kPa. The shear stress must exceed this value to create further dislocation loops. This stress value is very low, but evidently high enough to trigger dislocation movement under the applied high temperature conditions. The observation of single dislocation movement in a grain that shows no apparent distortion (i.e. homogeneous light grey image) underline the fact that it undergoes a nonnegligible local stress able to activate sources. The multiaxial stress acting on the dislocations may result from the applied temperature gradient, from the stress stored in the crystals during the growth process, and/or from the pressure of the crucible that acts on the crystals in the thickness direction. The presence of Frank-Read sources on regrown crystals have never been detected on films so far and show that a higher time resolution is definitely required for these time-dependent observations.

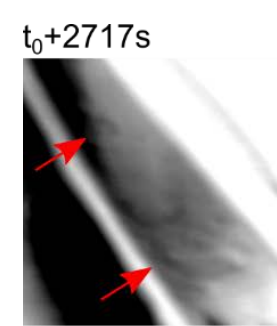

$\mathrm{T}_{\mathrm{T}}=1693 \mathrm{~K}$ $\mathrm{T}_{\mathrm{B}}=1543 \mathrm{~K}$
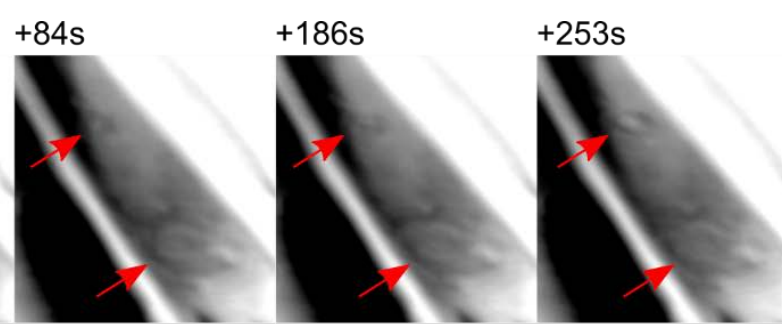

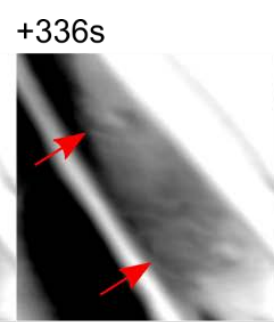

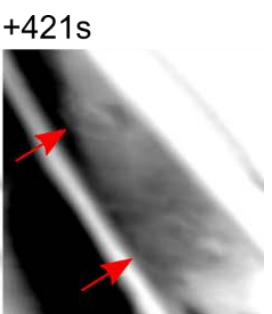

Figure 8: Topography image sequence showing one twin grain of silicon sample $B$ in a constant temperature gradient. Active Frank-Read sources are observed in the crystal about $1400 \mathrm{~s}$ after the start of solidification. $t_{0}$ corresponds to the starting time of solidification and $T_{T}$ and $T_{B}$ to the temperatures of the top and bottom heaters, respectively. A video is available in the supplementary material.

\section{Conclusion}

A real-time imaging setup for high-temperature crystal observations combining X-ray radiography and diffraction topography imaging is now available at beamline ID19 at the European Synchrotron Radiation Facility. This X-ray synchrotron imaging characterisation is coupled to the GaTSBI device that allows controlling the temperature gradient in the sample during heating, melting, solidification 
and cooling down at temperatures up to $2073 \mathrm{~K}$. The successful implementation of two cameras allows for synchronised and simultaneous image recording with acquisition rates of $0.5 \mathrm{~Hz}$ and spatial resolutions of up to $9 \mu \mathrm{m}$. Thus, the development of structural defects can be linked to the morphology of the solid-liquid interface. The measurements provide comprehensive insights into the high-temperature evolution of crystals, which is essential for the understanding of the growth processes to develop growth engineering techniques. Indeed, $\mathrm{X}$-ray radiography and topography are complementary tools in the characterisation of the dynamics of crystal growth. The X-ray radiography method provides information on the morphology and kinetics of the solid-liquid interface during melting and solidification. The X-ray Bragg diffraction topography provides information on the evolution of the grain shape and structure, the defect formation and the local level of crystal lattice distortion. The recent progress enables the synchronisation between topography and radiography images which offers the possibility to investigate the relationship between new grain nucleation and strain fields, which is important to identify crystal growth mechanisms. The increased acquisition rate of the topographs has proven to be particularly valuable in tracking dislocation movement and their interaction throughout the experiment.

We have presented experimental results to demonstrate how the method can contribute to a better understanding of several aspects occurring during crystal processing. This includes dislocation generation and motion in the seed crystal at high temperature up to the melting point as well as dislocation multiplication and rearrangement during melting, solidification and cooling. Moreover, dislocation propagation from the seed in the new grown crystal and along with the solid-liquid interface and their interaction with grain boundaries can be characterised. In the future, further experiments will be conducted to develop and deepen the investigation of these phenomena.

The discussion about the recording techniques makes it clear that a further improvement of the setup would require to record the evolution of several diffraction spots as they contain different and complementary information according to the corresponding crystallographic planes. An additional camera would provide more complete information on the anisotropy of the processes of growth, competition, dislocation interactions and multiplication. So, a first step would be to implement a third camera to record a second topography image. The implementation of an additional camera would be ideal to avoid the disadvantage of missing information by only recording one spot. However, the synchronisation of three cameras while maintaining the recording frequency is challenging, but attempts are being made to realise this in future campaigns. Another improvement path would consist in decreasing the scintillator thickness of the indirect detector system while increasing the spatial resolution to avoid blurring and improve the spatial resolution of the images. This would allow detecting finer features around the dislocations. 
Acknowledgements The team would like to express its debt to Dr. José Baruchel presently emeritus researcher at ESRF for constant support and most valuable advice for the realisation and analysis of these experiments throughout the project and since its very beginning.

\section{References}

Chikawa, J. I. \& Fujimoto, I. (1968). Applied Physics Letters 13, 387-389.

Danilewsky, A., Wittge, J., Kiefl, K., Allen, D., McNally, P., Garagorri, J., Elizalde, M. R., Baumbach, T. \& Tanner, B. K. (2013). Journal of applied crystallography 46, 849-855.

Danilewsky, A. N., Rack, A., Wittge, J., Weitkamp, T., Simon, R., Riesemeier, H. \& Baumbach, T. (2008). Nuclear Instruments and Methods in Physics Research Section B: Beam Interactions with Materials and Atoms 266, 2035-2040.

Danilewsky, A. N., Wittge, J., Croell, A., Allen, D., McNally, P., Vagovič, P., dos Santos Rolo, T., Li, Z., Baumbach, T., Gorostegui-Colinas, E., Garagorri, J., Elizalde, M. R., Fossati, M. C., Bowen, D. K. \& Tanner, B. K. (2011). Journal of Crystal Growth 318, 1157-1163.

Duffar, T. (2010). Recent Research Developments in crystal growth. 5, 61-111.

Fedotov, A., Evtodyi, B., Fionova, L., Ilyashuk, Y., Katz, E. \& Polyak, L. (1990). Phys Status Solidi A 119, 523-534.

George, A. (1977). Étude par tomographie aux rayons x de la vitesse de glissement des dislocations dans le silicium. Thèse d'Etat, INPL Nancy.

Hänschke, D., Danilewsky, A., Helfen, L., Hamann, E. \& Baumbach, T. (2017). Physical review letters 119, 215504.

Hartmann, W., Markewitz, G., Rettenmaier, U. \& Queisser, H. J. (1975). Applied Physics Letters 27, 308-309.

Oliveira, V. A., Sio, H. C., Faujour, A., Piot, L., Chabli, A. \& Camel, D. (2016). Energy Procedia 92, 755-763.

Oriwol, D., Carl, E. R., Danilewsky, A. N., Sylla, L., Seifert, W., Kittler, M. \& Leipner, H. S. (2013). Acta Materialia 61, 6903-6910.

Oriwol, D., Trempa, M., Sylla, L. \& Leipner, H. S. (2017). Journal of Crystal Growth 463, 1-9.

Rack, A., Garcia-Moreno, F., Schmitt, C., Betz, O., Cecilia, A., Ershov, A., Rack, T., Banhart, J. \& Zabler, S. (2010). Journal of X-ray science and technology 18, 429-441.

Rack, A., Scheel, M. \& Danilewsky, A. N. (2016). IUCrJ 3, 108-114.

Raghothamachar, B., Dhanaraj, G., Bai, J. \& Dudley, M. (2006). Microscopy research and technique 69, 343358.

Riberi-Béridot, T., Mangelinck-Noël, N., Tandjaoui, A., Reinhart, G., Billia, B., Lafford, T., Baruchel, J. \& Barrallier, L. (2015). Journal of Crystal Growth 418, 38-44.

Ryningen, B., Stokkan, G., Kivambe, M., Ervik, T. \& Lohne, O. (2011). Acta Materialia 59, 7703-7710.

Sopori, B., Rupnowski, P., Mehta, V., Budhraja, V., Johnston, S., Call, N., Mountinho, H., Al-Jassim, M., Shaikh, A., Seacrist, M. \& Carlson, D. (2009). 1049-1058.

Stamelou, V., Tsoutsouva, M. G., Riberi-Béridot, T., Reinhart, G., Regula, G., Baruchel, J. \& Mangelinck-Noël, N. (2017). Journal of Crystal Growth 479, 1-8.

Tandjaoui, A., Mangelinck-Noel, N., Reinhart, G., Billia, B. \& Guichard, X. (2013a). Comptes Rendus Physique 14, 141-148.

Tandjaoui, A., Mangelinck-Noel, N., Reinhart, G., Billia, B., Lafford, T. \& Baruchel, J. (2013b). Journal of Crystal Growth 377, 203-211.

Tandjaoui, A., Mangelinck-Noël, N., Reinhart, G., Furter, J. J., Billia, B., Lafford, T., Baruchel, J. \& Guichard, X. (2012). Energy Procedia 27, 82-87.

Tsoutsouva, M. G., Riberi-Béridot, T., Regula, G., Reinhart, G., Baruchel, J. \& Mangelinck-Noël, N. (2018). Physica Status Solidi (a) 215, 1700758.

Tsoutsouva, M. G., Riberi - Béridot, T., Regula, G., Reinhart, G., Baruchel, J., Guittonneau, F., Barrallier, L. \& Mangelinck-Noël, N. (2016). Acta Materialia 115, 210-223.

Tuomi, T., Naukkarinen, K. \& Rabe, P. (1974). Physica Status Solidi (a) 25, 93-106.

Usami, N., Yokoyama, R., Takahashi, I., Kutsukake, K., Fujiwara, K. \& Nakajima, K. (2010). Journal of Applied Physics 107, 013511.

Vallino, F., Chateau, J. P., Jacques, A. \& George, A. (2001). Mat Sci Eng a-Struct 319, 152-155.

Wang, Z.-J., Tsurekawa, S., Ikeda, K., Sekiguchi, T. \& Watanabe, T. (1999). Interface Sci 7, 197-205.

Wierzchowski, W., Wieteska, K., Graeff, W., Pawłowska, M., Surma, B. \& Strzelecka, S. (2004). Journal of Alloys and Compounds 362, 301-306.

Woo, S., Bertoni, M., Choi, K., Nam, S., Castellanos, S., Powell, D. M., Buonassisi, T. \& Choi, H. (2016). Solar Energy Materials and Solar Cells 155, 88-100. 
Wortman, J. J. \& Evans, R. A. (1965). Journal of Applied Physics 36, 153-156. 
Supporting information

\section{S1. Dislocation movement}

The video shows dislocation propagation during heating of sample $B$. The temperature is close to the melting point of silicon.

\section{Frank-Read sources}

The video shows the activation of Frank-Read sources on a regrown crystal in sample B during holding in a temperature gradient at high temperatures. 